\title{
Dynamic MRI for articulating joint evaluation on 1.5 T and 3.0 T scanners: setup, protocols, and real-time sequences
}

\author{
Marc Garetier ${ }^{1,2,3^{*}}$, Bhushan Borotikar ${ }^{3,4,5}$, Karim Makki ${ }^{3,6}$, Sylvain Brochard ${ }^{3,4,7,8}$, François Rousseau ${ }^{3,6}$ and
} Douraïed Ben Salem $3,4,9$

\begin{abstract}
Dynamic magnetic resonance imaging (MRI) is a non-invasive method that can be used to increase the understanding of the pathomechanics of joints. Various types of real-time gradient echo sequences used for dynamic MRI acquisition of joints include balanced steady-state free precession sequence, radiofrequency-spoiled sequence, and ultra-fast gradient echo sequence. Due to their short repetition time and echo time, these sequences provide high temporal resolution, a good signal-to-noise ratio and spatial resolution, and soft tissue contrast. The prerequisites of the evaluation of joints with dynamic MRI include suitable patient installation and optimal positioning of the joint in the coil to allow joint movement, sometimes with dedicated coil support. There are currently few recommendations in the literature regarding appropriate protocol, sequence standardizations, and diagnostic criteria for the use of real-time dynamic MRI to evaluate joints. This article summarizes the technical parameters of these sequences from various manufacturers on 1.5 T and 3.0 T MRI scanners. We have reviewed pertinent details of the patient and coil positioning for dynamic MRI of various joints. The indications and limitations of dynamic MRI of joints are discussed.
\end{abstract}

Keywords: Joints, Motion, Musculoskeletal system, Magnetic resonance imaging

\section{Key points}

- A real-time gradient echo sequence depicts functional details of the joint during motion.

- Appropriate and customized patient setup and coil installation inside the MR bore are fundamental for the exploration of joint motion.

- Artifacts due to the inherent joint motion and those related to real-time sequence parameters can be reduced to improve image quality and diagnostic capability.

\footnotetext{
* Correspondence: marc.garetier@wanadoo.fr

'Department of Radiology, Military Teaching Hospital Clermont-Tonnerre, Rue du colonel Fonferrier, 29240 Brest, Cedex 9, France

2Department of Radiology, University Hospital Morvan, Brest, France

${ }^{3}$ Laboratory of Medical Information Processing (LATIM), INSERM-UMR 1101,

Brest, France

Full list of author information is available at the end of the article
}

\section{Background}

Being non-invasive, magnetic resonance imaging (MRI) is widely used in the clinical decision-making process. Static morphological MRI is useful for the diagnosis of musculoskeletal disorders but does not represent the dynamic physiology of joints [1-5]. Dynamic in vivo imaging may provide valuable functional information (qualitative and quantitative) in addition to static imaging; thus, it may help in the selection of an optimal treatment strategy [6]. In vivo imaging of the joint motion can be performed using ultrasonography [7], single or biplanar fluoroscopy $[1,8]$, computed tomography (CT) [9], and MRI [10]. The role of ultrasonography is limited to the evaluation of the soft tissue around the joint. Fluoroscopy and CT scan modalities are limited to the quantification of bone kinematics and expose the individuals to ionizing radiation. MRI provides anatomical

\section{Springer Open}

(c) The Author(s). 2020 Open Access This article is licensed under a Creative Commons Attribution 4.0 International License, which permits use, sharing, adaptation, distribution and reproduction in any medium or format, as long as you give appropriate credit to the original author(s) and the source, provide a link to the Creative Commons licence, and indicate if changes were made. The images or other third party material in this article are included in the article's Creative Commons licence, unless indicated otherwise in a credit line to the material. If material is not included in the article's Creative Commons licence and your intended use is not permitted by statutory regulation or exceeds the permitted use, you will need to obtain permission directly from the copyright holder. To view a copy of this licence, visit http://creativecommons.org/licenses/by/4.0/. 
details of bones and soft tissues in static and dynamic acquisitions without exposure to ionizing radiation.

Dynamic MRI sequences were developed as early as 1984 for cardiac imaging $[11,12]$ and were subsequently applied to the musculoskeletal system to quantify bone motion and joint kinematics [13-15]. Dynamic MRIbased musculoskeletal system evaluations could be significantly different from those performed using static MRI. For example, Muhle et al. showed that dynamic MRI was significantly better than static imaging for the demonstration of patellar tilt angle, particularly at the critical range of patellar instability between $30^{\circ}$ and $0^{\circ}$ of knee flexion [16]. The existing literature shows no consensus regarding the technical parameters and the use of various sequences for dynamic MRI of joints. This is due to the existence of multiple and custom-built dynamic MRI sequences and the lack of standard/built-in dynamic musculoskeletal MRI sequences from the MRI scanners' original equipment manufacturers. Dynamic MRI is currently still not used in standard clinical practice for the management of musculoskeletal disorders [17]. Optimal dynamic MRI of the joint requires the following:

1) The adaptation of available dynamic MRI sequences to the shortest acquisition time to enable in vivo imaging of the joint during a single cycle of voluntary motion performed by the patient.

2) The customization of the MRI sequences and the scanning parameters according to the field strength and manufacturer of the MRI scanners.

3) The standardization of the patient setup and radiofrequency $(\mathrm{RF})$ coil positioning for each type of joint and its range of motion (ROM).

As many as eight types of dynamic MRI sequences have been reported in the literature. Of these, real-time sequences provide fast, trigger-free, and multi-slice acquisitions and are most suitable for in vivo joint evaluations in individuals with joint disorders [17]. In the following sections, we focus on real-time sequences and provide relevant details of the following:

1) Real-time sequence parameters used by multiple manufacturers on $1.5 \mathrm{~T}$ and $3.0 \mathrm{~T}$ MRI scanners.

2) Practical recommendations of patient and coil positioning in the scanner for optimal motion and image acquisition.

3) Perspectives and limitations on the use of dynamic MRI as standard clinical practice.

Real-time dynamic MRI sequences and parameters Joint motion imaging in MRI can be acquired by three methods [18]:
- Incremental (quasi-static) acquisition, with a change in the joint position between each acquisition. In this case, it is possible to use the static sequences, with the acquisition time multiplied by the number of sequence repetitions at each position [19-22].

- Motion-triggered acquisition, with the image database reconstructed according to the position of the joint during the acquisition cycle, requiring to repeat the movement several times, including cineMRI and cine phase-contrast techniques [23, 24].

- Real-time acquisition, allowing acquisition in a few seconds during continuous joint motion, with no repetition required [25-27].

In their systematic review, Borotikar et al. concluded that cine phase-contrast and real-time sequences were the two types of sequences that provided excellent validity and reliability for joint motion evaluation by MRI [17]. Cine phase-contrast sequences provide quantitative data, such as the three-dimensional (3D) pixel velocity in moving structures, coupled with anatomical images. However, image acquisition through these triggered sequences requires the patient to perform repeated joint motions. This leads to an acquisition time of a few minutes, which generates pain and fatigability in patients and in turn introduces averaging error in the acquired images due to the loss of movement reliability [10].

Real-time dynamic MRI, on the other hand, is a fast imaging technique that is mainly based on rapid gradient echo sequences with a flip angle of less than $90^{\circ}$ and a significant reduction of the repetition time (TR) lower than the T2 relaxation time. The low TR is responsible for a residual transverse magnetization before the next RF pulse. These sequences are particularly adapted for dynamic joint MRI because of their high temporal resolution, thereby allowing the acquisition of an image in a few hundred milliseconds and the fast repetition of the slices. The entire joint can be covered during a single acquisition, and the relationship between joint structures can be visualized to study normal and pathological joint physiology [26]. Three gradient echo sequences typically available on MRI scanners are suitable for real-time dynamic MRI and have already been used in previous studies to understand in vivo joint biomechanics. These are balanced steady-state free precession (SSFP) sequence [26, 28-33], RF-spoiled sequence [29, 34-36], and ultrafast gradient echo (UFGE) sequence [5, 37], with different acronyms used by each MRI scanner manufacturer (Table 1).

In a balanced SSFP sequence, a steady state between residual transverse and longitudinal magnetization is reached after the administration of a train of RF pulses, with the shortest TR and echo time (TE). All the gradients are fully refocused and balanced symmetrically, 
Table 1 Manufacturer acronyms of gradient echo sequences used for real-time joint dynamic MRI

\begin{tabular}{llll}
\hline & Balanced SSFP & RF-spoiled & UFGE \\
\hline GE Healthcare & FIESTA & SPGR & FSPGR \\
Philips Healthcare & bFFE & T1FFE & TFE \\
Siemens Healthineers & TrueFISP & FLASH & TurboFLASH
\end{tabular}

bFFE balanced Fast Field Echo, FLASH Fast Low-Angle SHot, FIESTA Fast Imaging Employing STeady-state Acquisition, FISP Fast Imaging with Steadystate Precession, FSPGR Fast SPoiled Gradient-Recalled, TFE Turbo Fast Echo

thereby allowing an excellent contrast between structures with a high signal-to-noise ratio (SNR), which is independent of TR and less sensitive to motion artifacts $[26,38-40]$. This sequence is T2-weighted, with contrast dependent on the T2/T1 ratio of each tissue, which results in a high fat and water signal (Figs. 1 and 2, Movie 1,2) [41]. In the RF-spoiled sequence, the RF phase modulation at each cycle deletes the residual transverse magnetization for T1 contrast with a TR value usually around $20-30 \mathrm{~ms}$ $[38,40]$. The UFGE sequence, based on the RF-spoiled gradient echo technique using a small flip angle, allows for the reduction of TR below $10 \mathrm{~ms}$ and, thus, reduces acquisition time [40, 42]. A few studies used a post-excitation refocused gradient echo sequence with radial sampling, allowing a T2/T1 contrast and high temporal resolution. However, this sequence is a work-in-progress package only provided by one manufacturer and cannot be proposed in the current practice [43].

Table 2 provides a summary of the sequence parameters reported in the literature since 2010 for real-time dynamic MRI of joints of the limbs with $1.5 \mathrm{~T}$ and $3.0 \mathrm{~T}$ MRI scanners, based on commercially available balanced SSFP, RF-spoiled, and UFGE sequences, for their implementation in daily practice.

The selection of sequence parameters is a trade-off among temporal resolution, SNR, and spatial resolution.
For example, Boutin et al. obtained a temporal resolution of $475 \mathrm{~ms}$ with a pixel size of $0.94 \mathrm{~mm}$ [33], whereas Pierrart et al. obtained a temporal resolution of $285 \mathrm{~ms}$ with a pixel size of $1.6 \mathrm{~mm}$ [28]. These sequences must be acquired with the shortest TR and TE to reduce acquisition time as well as the inhomogeneity due to T2* effects on balanced SSFP sequences [26]. A small flip angle improves SNR and provides a proton density-weighted image for RF-spoiled and UFGE sequences (Figs. 3 and 4, Movie 3, 4) [40, 42]. Cartesian and radial sampling of the $k$-space were both used for real-time dynamic MRI. Radial sampling was particularly suitable for real-time RF-spoiled sequences at $3.0 \mathrm{~T}$ [29, 35], allowing an increase in temporal resolution without image quality deterioration with the use of constrained iterative reconstruction [29]. Such iterative reconstruction of real-time images often requires the addition of graphic processing units to the current MRI systems [45, 46]. However, Cartesian sampling is more available and simpler to use for image reconstruction.

All slices covering the joint can be acquired during a single time frame by using sequential or multi-slice techniques $[28,35]$. The total acquisition time will depend on the number of slices and time frames. For example, Boutin et al. obtained an acquisition time of $35 \mathrm{~s}$ with one slice and 60 time frames for a balanced SSFP sequence [33], whereas Clarke et al. obtained an acquisition time of $104 \mathrm{~s}$ with eight slices and 40 time frames for a UFGE sequence [37]. Parameters such as the number of slices, gap, and field of view depend on the studied joint, patient size, and slice orientation.

The acquisition plane must be adapted to the joint being imaged. For example, on the ankle, an axial plane is more appropriate to evaluate fibular tendon instability [47], and a sagittal plane is more appropriate to evaluate Achilles tendon motion (Fig. 3, Movie 3) [48].

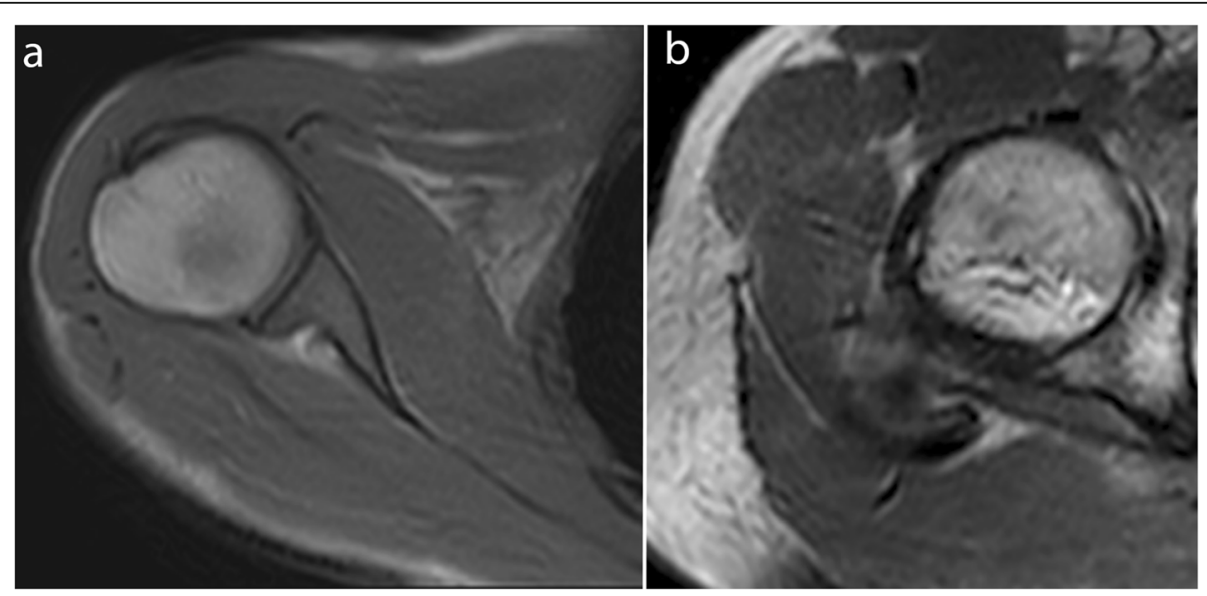

Fig. 1 Real-time dynamic MR images obtained with balanced SSFP sequence at 3.0 T. Shoulder in the axial plane in external rotation (a) and hip in the axial plane in external rotation (b) (TR, 4.2; TE, 2.1; flip angle, $40^{\circ}$; pixel size, $1.37 \times 1.83 \mathrm{~mm}$ ) 


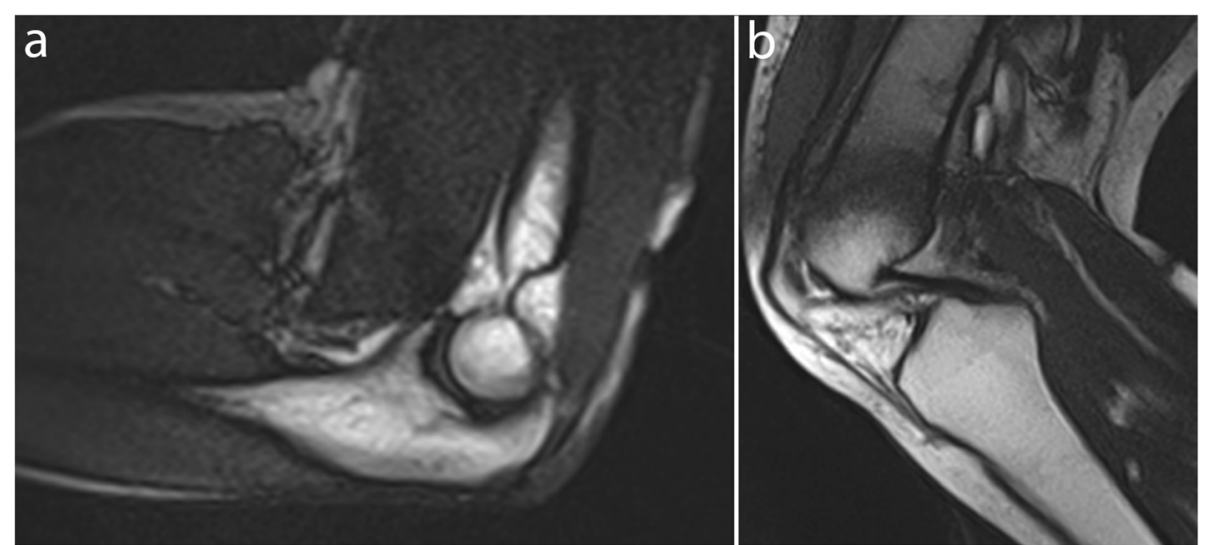

Fig. 2 Real-time dynamic MR images obtained with balanced SSFP sequence at 1.5 T. Elbow in the sagittal plane in flexion (a) and knee in the sagittal plane in flexion (b) (TR, 4.6; TE, 2.3; flip angle, $40^{\circ}$; pixel size, $1.09 \times 1.46 \mathrm{~mm}$ )

\section{Patient and radiofrequency coil positioning in the} MRI scanner

Optimal patient positioning in the scanner and RF coil positioning relative to the joint are fundamental for the accurate evaluation of joint motion with dynamic MRI. Joint and coil positioning inside the MRI scanner depends on the impairment and bone kinematics or tendon displacement to be explored (Table 3) (Movie 1, 2, 3 and 4).

The joint of interest must be positioned as centrally as possible inside the MRI bore to obtain a homogeneous field. Flex RF coils are often used for these acquisitions and must be positioned close to the joint for better signal homogeneity and SNR while preventing its displacement during joint motion. This may require specific fixtures to maintain the RF coil position close to the joint and to avoid contact with the body surface if necessary [26]. In most cases, cushions and devices provided by manufacturers should be effectively used without the need for additional fixtures [26, 32]. Joint positioning in the scanner requires a trade-off between the possible $\mathrm{ROM}$ and the necessity to be as close as possible to the
RF coil to enhance the SNR. This primarily depends on the RF coil type and size that is available for each scanner. Joint motion may also be limited by its positioning within the MRI bore and by the bore size on closed-bore MRI scanners.

The incorporation of dynamic MRI acquisition in addition to the standard clinical MR exam with static sequences poses multiple challenges. The position of the patient or the RF coil should not be changed throughout the exam. Furthermore, the addition of the dynamic sequence should not substantially increase the total acquisition time. The RF coils used for real-time dynamic MRI make it possible to acquire standard static images with high image quality and thus can be used for both static and dynamic acquisitions at the same time and with the same setup [5, 28, 29, 37].

Considering these requirements, we have provided recommendations from the literature for setting up each joint type in the MRI scanner. For this purpose, we classified the joints into three groups for patient positioning and equipment setup description:

Table 2 Sequence parameters reported in the recent literature for real-time joint dynamic MRI sequences at $1.5 \mathrm{~T}$ and $3.0 \mathrm{~T}$

\begin{tabular}{|c|c|c|c|c|c|}
\hline \multirow[b]{2}{*}{ Field strength } & \multicolumn{2}{|c|}{ Balanced SSFP } & \multicolumn{2}{|l|}{ RF-spoiled } & \multirow{2}{*}{$\begin{array}{l}\text { UFGE } \\
3.0 T[5,37]\end{array}$} \\
\hline & $1.5 \mathrm{~T}[28]$ & $3.0 \mathrm{~T}[29,33]$ & $1.5 \mathrm{~T}[36,44]$ & 3.0 T $[29,34,35]$ & \\
\hline TR (ms) & 3.6 & $3.98-4.71$ & $3.18-7.8$ & $3.13-20.6$ & $2.4-2.7$ \\
\hline TE (ms) & 1.3 & $1.75-2.36$ & $1.4-3.3$ & $1.74-3.3$ & $1.2-1.3$ \\
\hline Flip angle (degree) & 65 & $46-47$ & $8-20$ & $8-20$ & $10-15$ \\
\hline Bandwidth (Hz/pixel) & NR & 454-930 & 200, NR for [44] & 990 & NR \\
\hline Pixel size (mm) & 1.6 & $0.94-1.07$ & $1.4-1.56 \times 2.88$ & $0.57-1.07$ & 1 \\
\hline Slice thickness (mm) & 10 & 6 & 10 & $6-8$ & $4-5$ \\
\hline Number of time frames & 7 & $10-60$ & 40, NR for [44] & $10-16$ & $30-40$ \\
\hline Mean acquisition time/image (ms) & 285 & $475-562$ & $331-500$ & $197-351$ & $31-233$ \\
\hline
\end{tabular}




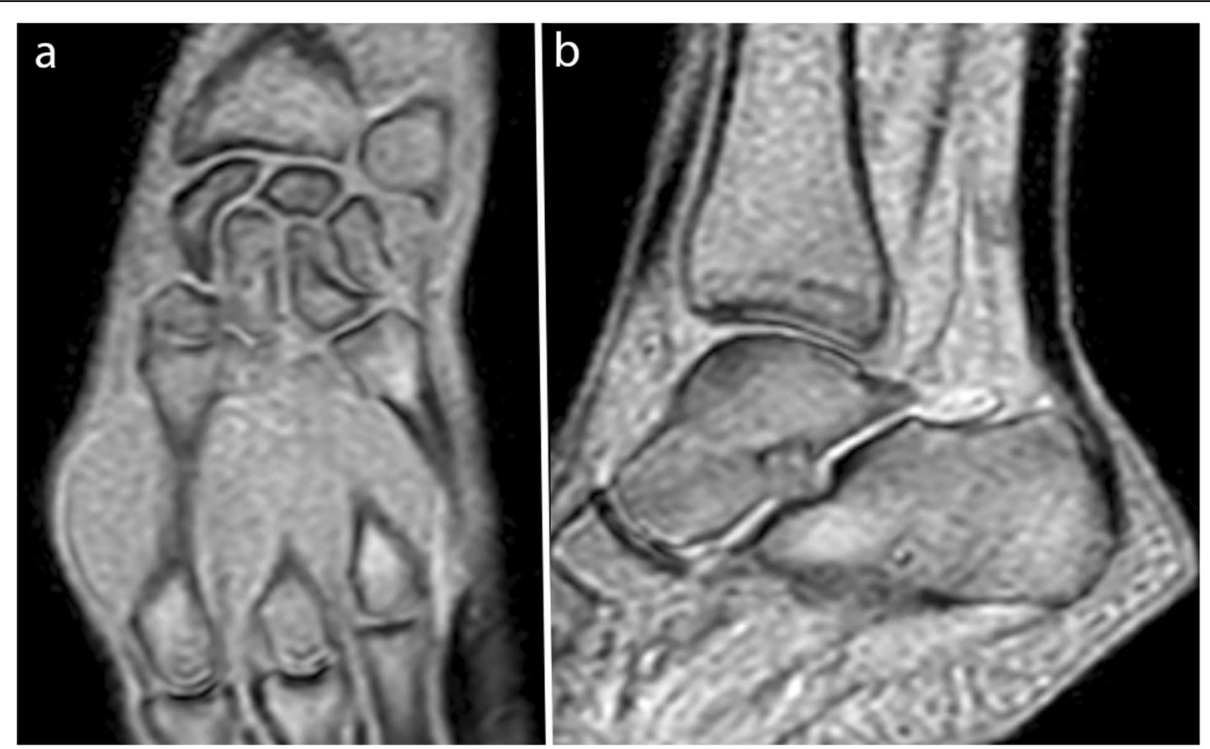

Fig. 3 Real-time dynamic MR images obtained with the RF-spoiled sequence at 3.0 T. Wrist in the coronal plane in ulnar deviation (a) and ankle in the sagittal plane in plantar flexion (b) (TR, 20.6; TE, 1.8; flip angle, $15^{\circ}$; pixel size, $1.3 \times 1.67 \mathrm{~mm}$ )

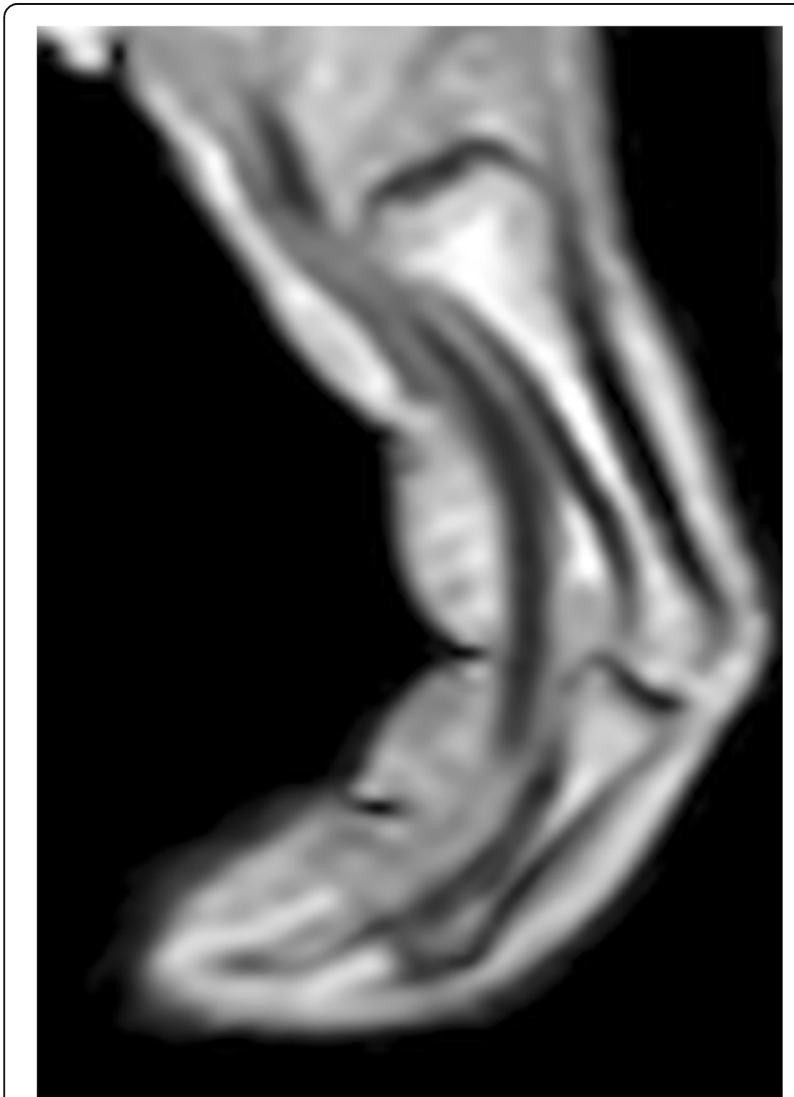

Fig. 4 Real-time dynamic MR image obtained with the UFGE sequence at 3.0 T. Finger in the sagittal plane in flexion (TR, 4.7; TE, 2.3; flip angle, $15^{\circ}$; pixel size, $1.09 \times 1.46 \mathrm{~mm}$ )
- Proximal joints (shoulder, hip)

- Intermediate joints (elbow, knee)

- Distal joints (wrist, hand, ankle, foot)

\section{Proximal joints}

Dynamic imaging of proximal joints is challenging because these joints are hard to reach for RF coil positioning. The setup should be optimized for achieving maximal ROM within the MRI scanner bore. The patient is typically positioned supine, head-first into the bore for the shoulder examination and feet-first for the hip examination, with the joint close to the MR bore center, which allows more space for movement to occur [28]. For the shoulder examination, the arm is positioned along the patient's side at rest [28, 58]. The coils are placed around the joint and could be maintained by a harness to avoid displacement during motion (Fig. 5, Movie 5) [58].

Table 3 Clinical applications of joint dynamic MRI

\begin{tabular}{ll}
\hline Shoulder & Subacromial impingement [49, 50] \\
\hline Wrist & Scapholunate instability [51] \\
& Extensor carpi ulnaris tendon instability [32] \\
Finger & Pulley injuries [52] \\
Hip & Femoroacetabular impingement [53] \\
Knee & Patellofemoral instability [16, 23, 43, 54] \\
& Anterior cruciate ligament deficiency [55-57] \\
& Post-traumatic medial laxity [36] \\
Ankle & Peroneal tendon subluxation [47] \\
\hline
\end{tabular}




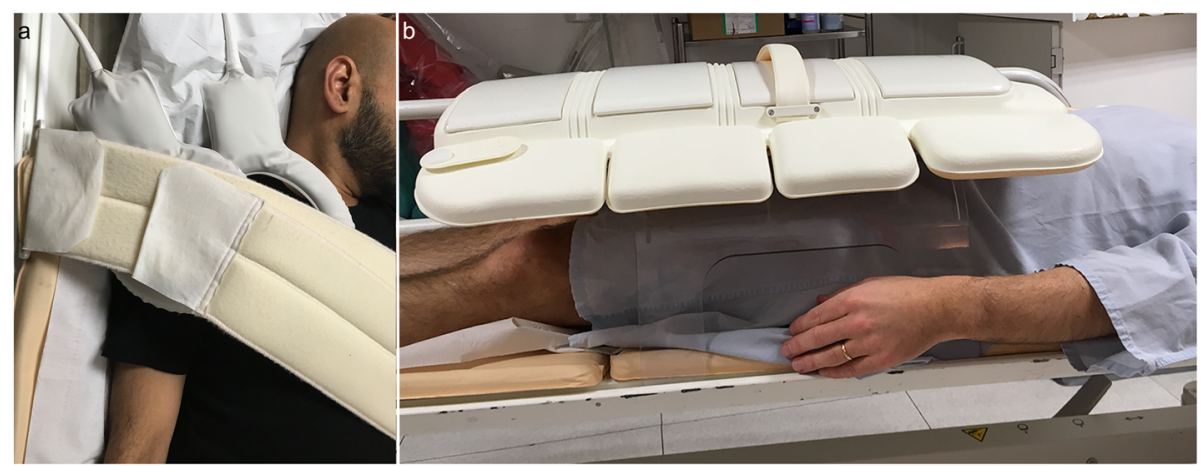

Fig. 5 Patient and coil positioning for shoulder (a) and hip (b) motion evaluation in an Achieva dStream 3.0T Philips MRI scanner

\section{Intermediate joints}

For the study of the elbow, the patient is positioned prone, head-first, arm above the head, with the elbow in the center of the RF coil which is held by a support (Fig. 6a) [26]. The kinematic study of the knee joint can be performed on a subject in a supine position, feet-first, with the flex coil held by a device around the joint (Fig. 6b) [59, 60]. The installation of a cushion under the knee increases the flexion/extension and degrees of freedom [5, 43], which are limited by the bore diameter and the size of the lower limb [61]. Unrestrained knee flexion/extension can also be achieved with the patient in side lying position and a large flex coil placed on the knee, and the other limb put on the coil to prevent its displacement (Fig. 6c) [44]. Some authors have also proposed prone positioning of the patient, allowing passive knee flexion [61] or knee flexion against resistance $[18,62]$.

\section{Distal joints}

All degrees of motion of distal joints can be studied within the MRI scanner. For the wrist and hand, the patient is positioned prone, head-first, with the upper limb raised above the head. Langner et al. studied the abduction/adduction motion of the wrist with a flex coil positioned on the table parallel to the motion without any support [51].

The distal situation of these joints makes it possible to use a "rigid" RF coil. Bayer et al. performed dynamic MRI sequences of the finger with a sky boot-shaped coil [63], whereas Schellhammer and Vantorre used a knee coil to study finger motion [52]. The hand can also be positioned within a head coil to investigate all degrees of freedom (Fig. 7a) [29, 33], whereas Kaiser et al. used an extremity coil to explore wrist pronation-supination [32]. Given the difference between the coil size and the small joint volume, the use of pads or foams to hold the hand in a central position within the coil and to avoid undesired motion is required (Fig. 7b) [32].

For the ankle, the patient is in the supine position, and images can be obtained using a flex coil that is positioned around the joint with a device adapted to the RF coil model (Fig. 7c) [22, 24, 26].

\section{Limitations, solutions, and perspectives}

Real-time dynamic MRI is based on high temporal resolution, but it also requires sufficient contrast, SNR, and spatial resolution for joint motion evaluations through image post-processing. The image quality also depends

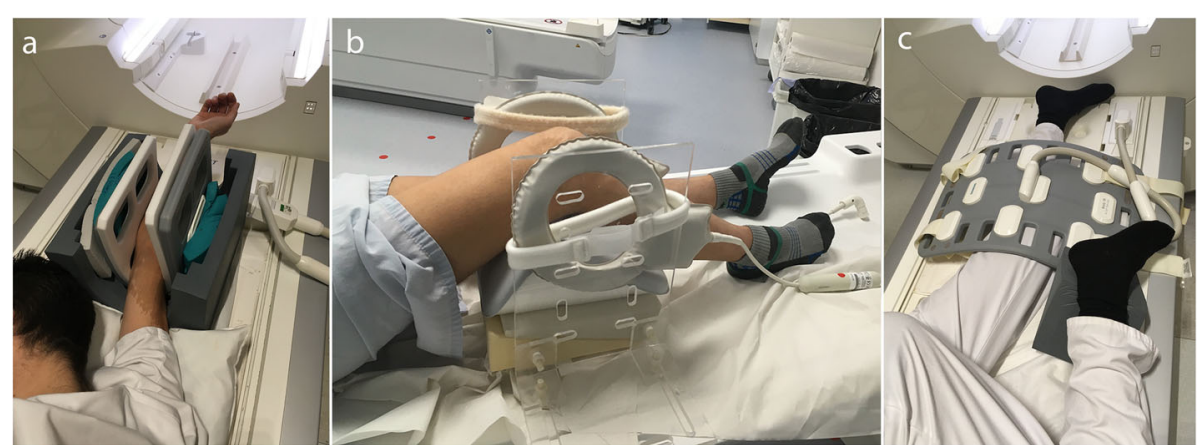

Fig. 6 Patient and flex coil positioning for elbow (a) and knee motion evaluation (b, c). The flex coil is held by a dedicated support in an Avanto fit 1.5 T Siemens MRI scanner for elbow motion evaluation (a). The patient is positioned supine with a homemade support for coil positioning in an Achieva dStream 3.0 T Philips MRI scanner (b) or lateral decubitus in an Avanto fit 1.5 T Siemens MRI scanner (c) for knee motion evaluation 

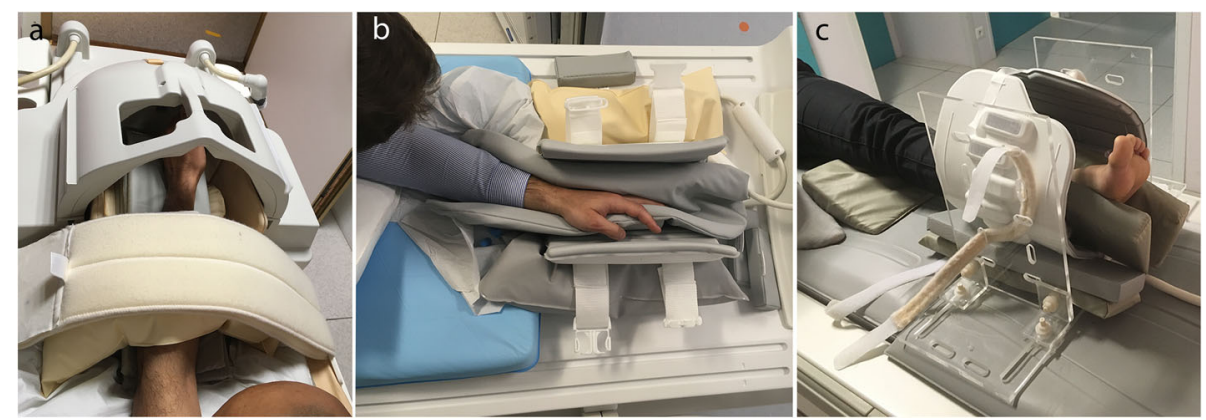

Fig. 7 Patient and coil positioning for wrist (a), finger (b), and ankle motion evaluation (c). The wrist is positioned within a head coil in an Achieva dStream 3.0 T Philips MRI scanner (a). The hand is positioned within an extremity coil (which is opened to show positioning inside) for finger motion evaluation in an Achieva dStream 3.0 T Philips MRI scanner (b). The flex coil is positioned in the support of ankle motion evaluation in an Optima 1.5 T GE (c). Some cushions and sandbags are added to maintain the joint in the selected plane

on the MRI scanner, magnetic field strength, and RF coil type [10]. Acquiring MRI at $3.0 \mathrm{~T}$ using flex coils with 16 channels can improve the acquisition time, SNR, and spatial resolution (Table 2).

However, these gradient echo sequences can be affected by some artifacts that worsen with higher magnetic field strength. Moreover, these sequences are susceptible to magnetic field inhomogeneities, resulting in signal loss and deformation. For example, a chemical shift artifact can appear as a black border at the fat/water interface. These artifacts can be reduced with a larger bandwidth, even if it decreases the SNR [33]. Chemical shift artifacts can also be reduced with in-phase TE [64].

Susceptibility and motion artifacts can be reduced with acceleration techniques such as parallel imaging with phased-array RF coils and partial or radial sampling of the $k$-space with iterative reconstructions [29, 65-68], which allow improvements in image quality and temporal resolution.

The balanced SSFP sequence is also deteriorated by band artifacts due to off-resonance effects from $B_{0}$ nonuniformity, which are not present in RF-spoiled and UFGE sequences. These band artifacts can appear over the joint and disturb image analysis, in particular at 3.0 $\mathrm{T}$ (Fig. 8) [29, 45]. They can be reduced by minimizing TR or by using a 3D shim at the beginning of the examination $[26,64]$. Other methods to reduce these band artifacts include the use of alternating TR, which widens the space between these bands [69]; multiple-offset method; frequency modulation [70]; or the use of
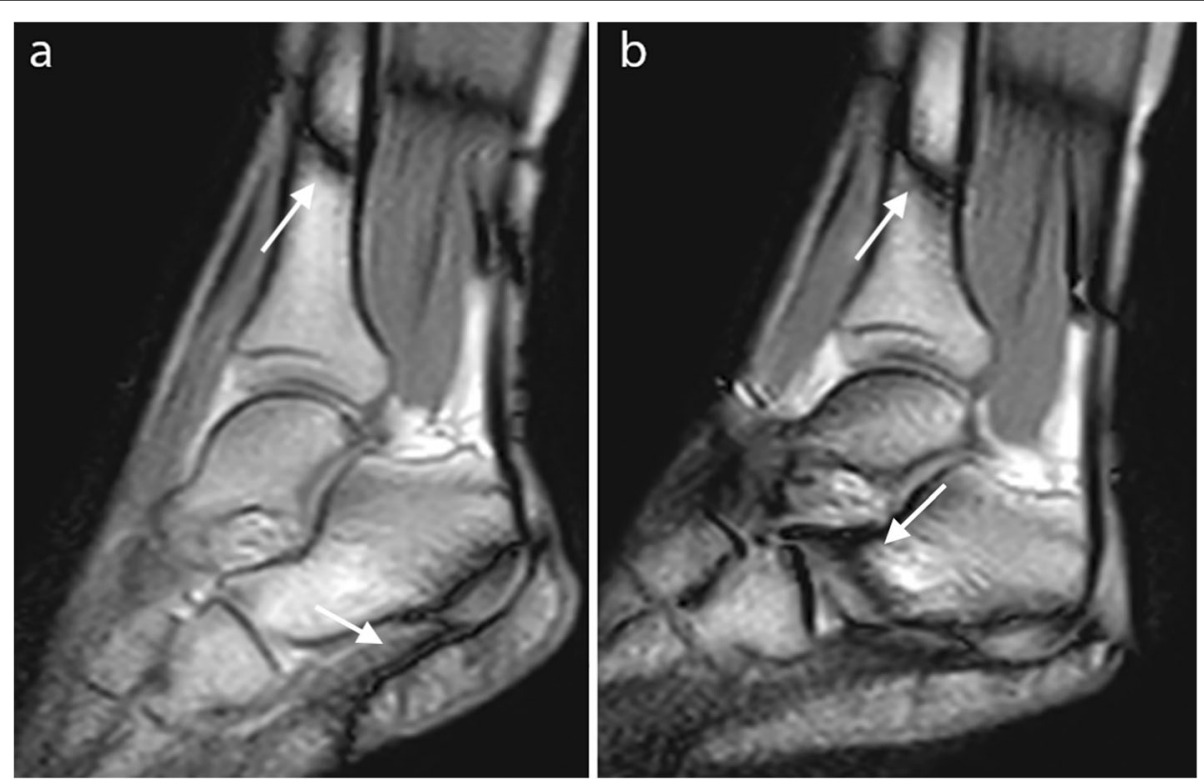

Fig. 8 Sagittal balanced SSFP image of the ankle in plantar (a) and dorsal (b) flexion. Band artifacts appear as black lines that move on the image during motion 
specific algorithms [71]. Band artifacts can also be reduced by positioning the joint in an intermediate position during calibration, considering its ROM [26]. Field inhomogeneity is increased in the case of large difference in size between the joint and the RF coil, as for finger examination. Adding dielectric pads around the joint can improve the homogeneity of the field and reduce artifacts [33].

New post-processing methods are being developed to register images obtained during motion with highresolution 3D static images. These methods make it possible to track bone motion in real-time [31,37], to obtain 3D reconstructions of the bone structures during motion [34], and, therefore, to provide accurate quantitative biomechanical data.

\section{Conclusion}

Three gradient echo sequences (balanced SSFP, RF-spoiled, and UFGE sequences) are available for real-time dynamic MRI of joints of the upper and lower limbs during continuous motion, considering their excellent temporal resolution, good SNR, and contrast. However, real-time dynamic MRI requires the adaptation of the sequence parameters, rigorous patient and coil positioning to allow an evaluation with a sufficient level of quality. These real-time sequences can be incorporated within a daily protocol for joint MR analysis due to their short acquisition time and would allow to better understand dynamic outcomes from specific joint disorders or to diagnose conditions not otherwise detected with static imaging. Future work may be focused on postprocessing method integration in current exams to improve the SNR and to obtain 3D reconstruction while maintaining a short acquisition time.

\section{Supplementary information}

Supplementary information accompanies this paper at https://doi.org/10. 1186/s13244-020-00868-5.

Additional file 1: Movie 1. Balanced SSFP sequence of the shoulder in the axial plane during rotation at 3.0 T (same parameters as Fig. 1). This sequence shows glenohumeral rotation, subscapular tendon excursion and the contraction of the subscapularis and infraspinatus muscles during internal and external rotation, respectively.

Additional file 2: Movie 2. Balanced SSFP sequence of the wrist in the coronal plane during radial/ulnar abduction at 3.0 T (same parameters as Fig. 1). This sequence shows wrist bones relationship during motion.

Additional file 3: Movie 3. RF-spoiled sequence of the ankle in the sagittal plane during flexion at 3.0 T (same parameters as Fig. 3). This sequence allows us to investigate tibiotalar motion and Achilles tendon excursion.

Additional file 4: Movie 4. UFGE sequence of the finger in the sagittal plane during flexion at 3.0 T (same parameters as Fig. 4). This sequence allows the evaluation of bones motion and flexor tendon excursion.

Additional file 5: Movie 5. Shoulder motion in the three degrees of freedom after coil positioning.

\section{Abbreviations}

3D: Three-dimensional; CT: Computed tomography; MRI: Magnetic resonance imaging; RF: Radio frequency; ROM: Range of motion; SNR: Signal-to-noise ratio; SSFP: Steady-state free precession; TE: Echo time; TR: Repetition time; UFGE: Ultra-fast gradient echo

\section{Acknowledgements}

The authors thank the application engineers from GE Healthcare, Philips Healthcare, and Siemens Healthineers for their valuable help, and the radiographers from Brest University and Military Teaching hospitals.

\section{Authors' contributions}

MG was the major contributor in writing and organizing the manuscript. MG and BB conducted the literature search. BB and DBS supervised the work and made substantial contributions to the design of the study. KM, SB, and FR read, reviewed, and contributed with their expertise in their different fields to the final manuscript. All authors read and approved the final manuscript.

Funding

The authors declare that this work has not received any funding.

Availability of data and materials

Not applicable

Ethics approval and consent to participate

Not applicable

\section{Consent for publication}

Not applicable

\section{Competing interests}

The authors declare that they have no competing interests.

\section{Author details}

${ }^{1}$ Department of Radiology, Military Teaching Hospital Clermont-Tonnerre, Rue du colonel Fonferrier, 29240 Brest, Cedex 9. France. ${ }^{2}$ Department of Radiology, University Hospital Morvan, Brest, France. ${ }^{3}$ Laboratory of Medical Information Processing (LATIM), INSERM-UMR 1101, Brest, France. ${ }^{4}$ University of Western Brittany (UBO), Brest, France. ${ }^{5}$ University Hospital, Brest, France. ${ }^{6}$ IMT Atlantique, UBL, Brest, France. ${ }^{7}$ Department of Physical and Medical Rehabilitation, University Hospital Morvan, Brest, France. ${ }^{8}$ Department of Paediatric Physical and Medical Rehabilitation, Fondation Ildys, Brest, France. ${ }^{9}$ Department of Radiology, University Hospital La Cavale Blanche, Brest, France.

Received: 23 November 2019 Accepted: 2 April 2020

Published online: 19 May 2020

\section{References}

1. Tashman S, Collon D, Anderson K, Kolowich P, Anderst W (2004) Abnormal rotational knee motion during running after anterior cruciate ligament reconstruction. Am J Sports Med 32:975-983

2. Draper CE, Besier TF, Fredericson M et al (2011) Differences in patellofemoral kinematics between weight-bearing and non-weight-bearing conditions in patients with patellofemoral pain. J Orthop Res 29:312-317

3. Powers CM, Ward SR, Fredericson M, Guillet M, Shellock FG (2003) Patellofemoral kinematics during weight-bearing and non-weight-bearing knee extension in persons with lateral subluxation of the patella: a preliminary study. J Orthop Sports Phys Ther 33:677-685

4. McWalter EJ, O'Kane CM, FitzPatrick DP, Wilson DR (2014) Validation of an MRI-based method to assess patellofemoral joint contact areas in loaded knee flexion in vivo: patellofemoral contact area validation. J Magn Reson Imaging 39:978-987

5. d'Entremont AG, Nordmeyer-Massner JA, Bos C, Wilson DR, Pruessmann KP (2013) Do dynamic-based MR knee kinematics methods produce the same results as static methods? Magn Reson Med 69:1634-1644

6. Carr R, MacLean S, Slavotinek J, Bain Gl (2019) Four-dimensional computed tomography scanning for dynamic wrist disorders: prospective analysis and recommendations for clinical utility. J Wrist Surg 08:161-167

7. Guillin R, Marchand AJ, Roux A, Niederberger E, Duvauferrier R (2012) Imaging of snapping phenomena. Br J Radiol 85:1343-1353 
8. Li G, Van de Velde SK, Bingham JT (2008) Validation of a non-invasive fluoroscopic imaging technique for the measurement of dynamic knee joint motion. J Biomech 41:1616-1622

9. Teixeira P, Gervaise A, Louis M et al (2015) Musculoskeletal wide-detector CT kinematic evaluation: from motion to image. Semin Musculoskelet Radiol 19:456-462

10. Shapiro LM, Gold GE (2012) MRI of weight bearing and movement. Osteoarthritis Cartilage 20:69-78

11. Sechtem U, Pflugfelder PW, White RD et al (1987) Cine MR imaging: potential for the evaluation of cardiovascular function. AJR Am J Roentgenol 148:239-246

12. van Dijk P (1984) ECG-triggered NMR imaging of the heart. Diagn Imaging Clin Med 53:29-37

13. Melchert UH, Schröder C, Brossmann J, Muhle C (1992) Motion-triggered cine MR imaging of active joint movement. Magn Reson Imaging 10:457460

14. Burnett KR, Davis CL, Read J (1987) Dynamic display of the temporomandibular joint meniscus by using "fast-scan" MR imaging. AJR Am J Roentgenol 149:959-962

15. Zhu Y, Pelc NJ (1999) Three-dimensional motion tracking with volumetric phase contrast MR velocity imaging. J Magn Reson Imaging 9:111-118

16. Muhle C, Brossmann J, Melchert UH et al (1995) Functional MRI of the patellofemoral joint: comparison of ultrafast MRI, motion-triggered cine MRI and static MRI. Eur Radiol 5:371-378

17. Borotikar B, Lempereur M, Lelievre M, Burdin V, Ben Salem D, Brochard S (2017) Dynamic MRI to quantify musculoskeletal motion: a systematic review of concurrent validity and reliability, and perspectives for evaluation of musculoskeletal disorders. PLoS One 12:e0189587

18. Shellock FG (2003) Functional assessment of the joints using kinematic magnetic resonance imaging. Semin Musculoskelet Radiol 7:249-276

19. Shellock FG, Mink JH, Deutsch AL, Fox JM (1989) Patellar tracking abnormalities: clinical experience with kinematic MR imaging in 130 patients. Radiology 172:799-804

20. Schmid MR, Hodler J, Cathrein P, Duewell S, Jacob HAC, Romero J (2002) Is impingement the cause of jumper's knee? Dynamic and static magnetic resonance imaging of patellar tendinitis in an open-configuration system. Am J Sports Med 30:388-395

21. Scarvell JM, Smith PN, Refshauge KM, Galloway HR, Woods KR (2005) Association between abnormal kinematics and degenerative change in knees of people with chronic anterior cruciate ligament deficiency: a magnetic resonance imaging study. Aust J Physiother 51:233-240

22. Tokuda O, Awaya H, Taguchi K, Matsunga N (2006) Kinematic MRI of the normal ankle ligaments using a specially designed passive device. Foot Ankle Int 27:935-942

23. Brossmann J, Muhle C, Büll CC et al (1994) Evaluation of patellar tracking in patients with suspected patellar malalignment: cine MR imaging vs arthroscopy. AJR Am J Roentgenol 162:361-367

24. Sheehan FT, Seisler AR, Siegel KL (2007) In vivo talocrural and subtalar kinematics: a non-invasive 3D dynamic MRI study. Foot Ankle Int 28:323-335

25. Shellock FG, Foo TK, Deutsch AL, Mink JH (1991) Patellofemoral joint: evaluation during active flexion with ultrafast spoiled GRASS MR imaging. Radiology 180:581-585

26. Quick HH, Ladd ME, Hoevel M et al (2002) Real-time MRI of joint movement with trueFISP. J Magn Reson Imaging 15:710-715

27. Draper CE, Santos JM, Kourtis LC et al (2008) Feasibility of using real-time MRI to measure joint kinematics in $1.5 \mathrm{~T}$ and open-bore $0.5 \mathrm{~T}$ systems. J Magn Reson Imaging 28:158-166

28. Pierrart J, Lefèvre-Colau MM, Skalli W et al (2014) New dynamic threedimensional MRI technique for shoulder kinematic analysis. J Magn Reson Imaging 39:729-734

29. Shaw CB, Foster BH, Borgese M et al (2019) Real-time three-dimensional MR for the assessment of dynamic carpal instability. PLoS One 14:e0222704

30. Yen P, Katzberg RW, Buonocore MH, Sonico J (2013) Dynamic MR imaging of the temporomandibular joint using a balanced steady-state free precession sequence at 3T. AJNR Am J Neuroradiol 34:E24-E26

31. Gilles B, Perrin R, Magnenat-Thalmann N, Vallee JP (2005) Bone motion analysis from dynamic MRI: acquisition and tracking. Acad Radiol 12:12851292

32. Kaiser P, Kellermann F, Arora R, Henninger B, Rudisch A (2018) Diagnosing extensor carpi ulnaris tendon dislocation with dynamic rotation MRI of the wrist. Clin Imaging 51:323-326
33. Boutin RD, Buonocore MH, Immerman I et al (2013) Real-time magnetic resonance imaging (MRI) during active wrist motion-initial observations. PLoS One 8:e84004

34. Makki K, Borotikar B, Garetier M, Brochard S, Ben Salem D, Rousseau F (2019) In vivo ankle joint kinematics from dynamic magnetic resonance imaging using a registration-based framework. J Biomech 86:193-203

35. Henrichon SS, Foster BH, Shaw C et al (2020) Dynamic MRI of the wrist in less than 20 seconds: normal midcarpal motion and reader reliability. Skeletal Radiol 49:241-248

36. Studler U, White LM, Deslandes M, Geddes C, Sussman MS, Theodoropoulos J (2011) Feasibility study of simultaneous physical examination and dynamic MR imaging of medial collateral ligament knee injuries in a 1.5-T large-bore magnet. Skeletal Radiol 40:335-343

37. Clarke EC, Martin JH, d'Entremont AG, Pandy MG, Wilson DR, Herbert RD (2015) A non-invasive, 3D, dynamic MRI method for measuring muscle moment arms in vivo: demonstration in the human ankle joint and Achilles tendon. Med Eng Phys 37:93-99

38. Chavhan GB, Babyn PS, Jankharia BG, Cheng H-LM, Shroff MM (2008) Steady-state MR imaging sequences: physics, classification, and clinical applications. Radiographics 28:1147-1160

39. Bieri O, Scheffler K (2013) Fundamentals of balanced steady state free precession MRI. J Magn Reson Imaging 38:2-11

40. Hargreaves B (2012) Rapid gradient-echo imaging. J Magn Reson Imaging 36:1300-1313

41. Fuchs F, Laub G, Othomo K (2003) TrueFISP_technical considerations and cardiovascular applications. Eur J Radiol 46:28-32

42. Elster AD (1993) Gradient-echo MR imaging: techniques and acronyms. Radiology 186:1-8

43. Burke CJ, Kaplan D, Block T et al (2018) Clinical utility of continuous radial magnetic resonance imaging acquisition at $3 \mathrm{~T}$ in real-time patellofemoral kinematic assessment: a feasibility study. Arthroscopy 34:726-733

44. Fiorentino NM, Lin JS, Ridder KB, Guttman MA, McVeigh ER, Blemker SS (2013) Rectus femoris knee muscle moment arms measured in vivo during dynamic motion with real-time magnetic resonance imaging. J Biomech Eng 135:044501

45. Lingala SG, Sutton BP, Miquel ME, Nayak KS (2016) Recommendations for real-time speech MRI. J Magn Reson Imaging JMRI 43:28-44

46. Frahm J, Voit D, Uecker M (2019) Real-time magnetic resonance imaging: radial gradient-echo sequences with nonlinear inverse reconstruction. Invest Radiol 54:757-766

47. VanPelt MD, Landrum MR, Igbinigie M, Wadhwa V, Chhabra A (2017) Kinematic magnetic resonance imaging of peroneal tendon subluxation with intraoperative correlation. J Foot Ankle Surg 56:395-397

48. Sheehan FT (2012) The 3D in vivo Achilles' tendon moment arm, quantified during active muscle control and compared across sexes. J Biomech 45:225-230

49. Tempelaere C, Pierrart J, Lefèvre-Colau MM et al (2016) Dynamic threedimensional shoulder MRI during active motion for investigation of rotator cuff diseases. PLoS One 11:e0158563

50. Tasaki A, Nimura A, Nozaki T et al (2015) Quantitative and qualitative analyses of subacromial impingement by kinematic open MRI. Knee Surg Sports Traumatol Arthrosc 23:1489-1497

51. Langner I, Fischer S, Eisenschenk A, Langner S (2015) Cine MRI: a new approach to the diagnosis of scapholunate dissociation. Skeletal Radiol 44:1103-1110

52. Schellhammer F, Vantorre A (2019) Semi-dynamic MRI of climbingassociated injuries of the finger. Skeletal Radiol 48:1435-1437

53. Burke CJ, Walter WR, Gyftopoulos S et al (2019) Real-time assessment of femoroacetabular motion using radial gradient echo magnetic resonance arthrography at 3 Tesla in routine clinical practice: a pilot study. Arthroscopy 35:2366-2374

54. Muhle C, Brossmann J, Heller M (1999) Kinematic CT and MR imaging of the patellofemoral joint. Eur Radiol 9:508-518

55. Guenoun D, Vaccaro J, Le Corroller T et al (2017) A dynamic study of the anterior cruciate ligament of the knee using an open MRI. Surg Radiol Anat 39:307-314

56. Haughom BD, Souza R, Schairer WW, Li X, Benjamin Ma C (2012) Evaluating rotational kinematics of the knee in ACL-ruptured and healthy patients using 3.0 Tesla magnetic resonance imaging. Knee Surg Sports Traumatol Arthrosc 20:663-670

57. Barrance PJ, Williams GN, Snyder-Mackler L, Buchanan TS (2006) Altered knee kinematics in ACL-deficient non-copers: a comparison using dynamic MRI. J Orthop Res 24:132-140 
58. Matsui K, Tachibana T, Nobuhara K, Uchiyama Y (2018) Translational movement within the glenohumeral joint at different rotation velocities as seen by cine MRI. J Exp Orthop 5:7

59. Borotikar BS, Sipprell WH 3rd, Wible EE, Sheehan FT (2012) A methodology to accurately quantify patellofemoral cartilage contact kinematics by combining 3D image shape registration and cine-PC MRI velocity data. J Biomech 45:1117-1122

60. Westphal CJ, Schmitz A, Reeder SB, Thelen DG (2013) Load-dependent variations in knee kinematics measured with dynamic MRI. J Biomech 46: 2045-2052

61. Lin CC, Zhang S, Frahm J, Lu TW, Hsu CY, Shih TF (2013) A slice-to-volume registration method based on real-time magnetic resonance imaging for measuring three-dimensional kinematics of the knee. Med Phys 40:102302

62. Powers CM, Shellock FG, Pfaff M (1998) Quantification of patellar tracking using kinematic MRI. J Magn Reson Imaging 8:724-732

63. Bayer T, Adler W, Janka R, Uder M, Roemer F (2017) Magnetic resonance cinematography of the fingers: a 3.0 Tesla feasibility study with comparison of incremental and continuous dynamic protocols. Skeletal Radiol 46:17211728

64. Huang SY, Seethamraju RT, Patel P, Hahn PF, Kirsch JE, Guimaraes AR (2015) Body MR imaging: artifacts, k-space, and solutions. Radiographics 35:14391460

65. Zaitsev M, Maclaren J, Herbst M (2015) Motion artifacts in MRl: a complex problem with many partial solutions. J Magn Reson Imaging 42:887-901

66. Deshmane A, Gulani V, Griswold MA, Seiberlich N (2012) Parallel MR imaging. J Magn Reson Imaging 36:55-72

67. Tsao J, Kozerke S (2012) MRI temporal acceleration techniques. J Magn Reson Imaging 36:543-560

68. Uecker M, Zhang S, Voit D, Merboldt KD, Frahm J (2012) Real-time MRl: recent advances using radial FLASH. Imaging Med 4:461-476

69. Nayak KS, Lee HL, Hargreaves BA, Hu BS (2007) Wideband SSFP: alternating repetition time balanced steady state free precession with increased band spacing. Magn Reson Med 58:931-938

70. Foxall DL (2002) Frequency-modulated steady-state free precession imaging Magn Reson Med 48:502-508

71. Björk M, Ingle RR, Gudmundson E, Stoica P, Nishimura DG, Barral JK (2014) Parameter estimation approach to banding artifact reduction in balanced steady-state free precession. Magn Reson Med 72:880-892

\section{Publisher's Note}

Springer Nature remains neutral with regard to jurisdictional claims in published maps and institutional affiliations.

\section{Submit your manuscript to a SpringerOpen ${ }^{\circ}$ journal and benefit from:}

- Convenient online submission

- Rigorous peer review

- Open access: articles freely available online

- High visibility within the field

- Retaining the copyright to your article

Submit your next manuscript at $\boldsymbol{\nabla}$ springeropen.com 